livraisons

d'Histoire

de l'Architecture

\section{Livraisons de l'histoire de l'architecture}

$22 \mid 2011$

Émotions patrimoniales II

\title{
Reconstruire le Palais des Tuileries. Une émotion patrimoniale et politique « rémanente »? (1871-2011)
}

The Reconstruction of the Tuileries Palace, a Reccurrent Political and Heritage Emotion (1871-2011)

Den Tuilerienpalast wiederaufbauen : eine remanente émotion patrimoniale (1871-2011)

\section{Vincent Lemire et Yann Potin}

\section{(2) OpenEdition}

\section{Journals}

Édition électronique

URL : http://journals.openedition.org/lha/293

DOI : 10.4000/lha.293

ISSN : 1960-5994

Éditeur

Association Livraisons d'histoire de l'architecture - LHA

Édition imprimée

Date de publication : 10 décembre 2011

Pagination : 87-108

ISSN : 1627-4970

Référence électronique

Vincent Lemire et Yann Potin, «Reconstruire le Palais des Tuileries. Une émotion patrimoniale et politique « rémanente »? (1871-2011)», Livraisons de l'histoire de l'architecture [En ligne], 22 | 2011, mis en ligne le 10 décembre 2013, consulté le 19 avril 2019. URL : http://journals.openedition.org//ha/293 ; DOl : $10.4000 /$ /ha. 293

Ce document a été généré automatiquement le 19 avril 2019

Tous droits réservés à l'Association LHA 


\title{
Reconstruire le Palais des Tuileries. Une émotion patrimoniale et politique « rémanente »? (1871-2011)
}

\author{
The Reconstruction of the Tuileries Palace, a Reccurrent Political and Heritage \\ Emotion (1871-2011) \\ Den Tuilerienpalast wiederaufbauen : eine remanente émotion patrimoniale \\ (1871-2011)
}

\section{Vincent Lemire et Yann Potin}

1 La commémoration du $140^{\mathrm{e}}$ anniversaire de la Commune de Paris, entre mars et mai 2011, a rencontré une certaine indifférence médiatique, malgré la tenue d'une exposition officielle au sein de l'Hôtel de Ville, qui par son existence et sa localisation même a constitué un événement : on pouvait y voir, en effet, une certaine forme de banalisation, sinon d'apaisement des mémoires. Sur le plan architectural, la Commune demeure cependant la source indirecte d'un traumatisme patrimonial, à la fois parisien et national, qui a pu étrangement renaître au cours de la dernière décennie.

2 Il y a près de 10 ans, en effet, le 18 décembre 2002, l'Académie du Second Empire organisait, avec le concours de l'Établissement public du grand Louvre, une journée de colloque visant à célébrer le cent cinquantenaire du décret présidentiel du 12 mars 1852, portant réunion des Tuileries au Louvre ${ }^{2}$. Par la voix de son président, Alain Boumier, cette association dédiée à la mémoire du «dernier souverain de France ", concluait la journée par un appel inattendu aux pouvoirs publics : reconstruire à l'identique le palais des Tuileries, incendié le 24 mai 1871 par la Commune puis démoli par la III République naissante suite à une loi votée en juin 1882. Il s'agissait ni plus ni moins que de remettre à l'ordre du jour l'achèvement du « Grand dessein » palatial français, qui depuis, Henri IV et jusqu'à Napoléon III, n'aurait cessé d'animer le cœur battant d'une centralité monarchique transfigurée par l'architecture classique des Philibert de l'Orme, des 
Androuet du Cerceau et des Le Vau. Malgré près de cent trente années de vide topographique, au cœur même de la capitale, la "présence ineffaçable» du lieu serait ainsi susceptible de commander la « rémanence de sa charge émotionnelle ».

Dès la fin mois d'octobre 2003, une exposition intitulée «Le Louvre demain, reconstruire les Tuileries", était inaugurée sous le patronage de l'association des grands prix de Rome d'architecture et des grands prix d'architecture de l'Académie des Beaux-arts, dans le beffroi néo-gothique de la mairie du $\mathrm{I}^{\mathrm{er}}$ arrondissement, face à la colonnade du Louvre. Une série de maquettes, représentant autant de projets de reconstruction possibles du palais disparu, semblait soudain rendre réalisable, et donc réel, ce qui n'était alors qu'une utopie, portée par un discours résolument nostalgique (ill. 1). Comment expliquer une si rapide issue ? La concordance des temps est troublante : au moment même où le schéma directeur du « grand Versailles » était adopté, le 30 octobre 2003, proposant, entre autres, la "restitution » " de la grille royale de la cour centrale du château, l'exposition de maquettes de Tuileries « restituées » semblait vouloir profiter de ce précédent en matière de politique patrimoniale, et qui, depuis lors, a effectivement été réalisé en 2008 par l'architecte Frédéric Didier. Jusqu'alors, et depuis la fin du XIX siècle, toute "restitution" d'éléments architecturaux disparus apparaissait autant comme une tentation permanente que comme un tabou professionnel et scientifique, ainsi que le rappelait en novembre 2001, à la veille même du "passage à l'acte » patrimonial que constitue la restitution de la grille de Versailles, un inspecteur des Monuments historiques, François Macé de l'Épinay, au cours d'une session des fameux Entretiens du patrimoines.

III. 1 : Vue du pavillon de l'Horloge, depuis le bassin central du jardin des Tuileries

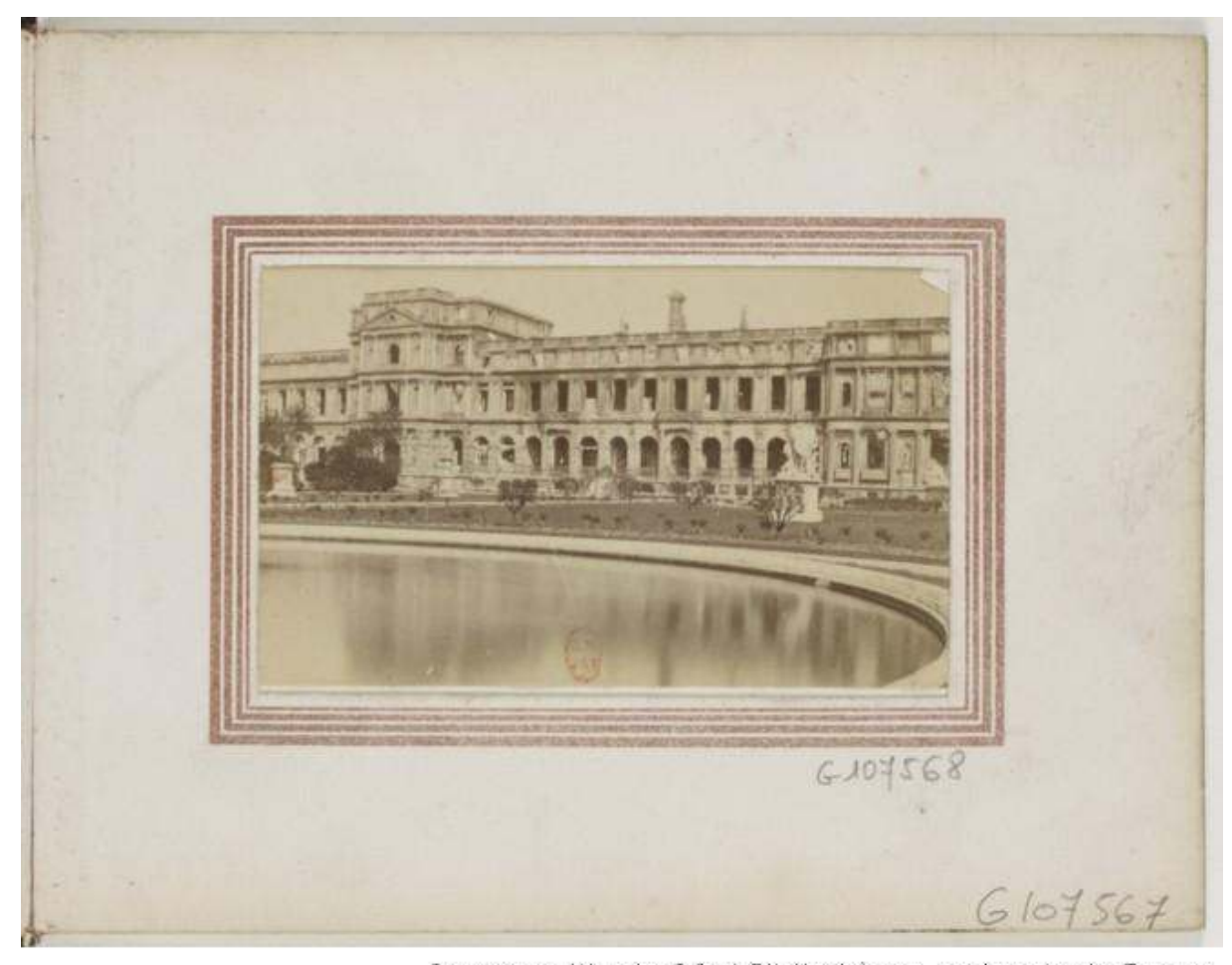

Source gallica.bnf.fr / Bibliothèque nationale de France

Publié dans Ruines de Paris, Mai 1871, Bibliothèque nationale de France, département Estampes et photographie, 8-VE-419

$\mathrm{Cl}$. Gallica.fr / Bibliothèque nationale de France 
4 À partir de l'exposition propitiatoire d'octobre 2003, on peut dire que l'émotion a suivi un chemin ascendant, mais que cette montée en puissance n'a pourtant pas rencontré une réelle embardée médiatique, bien que le Le Figaro ait choisi de faire du projet de reconstruction sa Une le 14 février 2004. Au titre quelque peu distancé de la première page - «Le projet fou des Tuileries» - répondait en page intérieure un bandeau plus prometteur, sous la plume d'Anne Muratori-Philipp : « La reconstruction des Tuileries en marche ». Ce n'est toutefois qu'en décembre 2004 que les statuts d'un " comité national pour la reconstruction des Tuileries », présidé par le même Alain Boumier, furent déposés en préfecture, sous la forme d'une association se proposant de n'obtenir de l'État qu'une autorisation administrative en vue de mener à bien un projet qui serait fondé sur un mécénat privé (ill. 2). Multipliant journées d'information, reportages médiatiques et conférences de presse, le comité parvint à obtenir du ministère de la Culture, en juin 2006, la création d'une commission d'études, avant de susciter l'adhésion au comité de plus de 1500 personnes, provenant de milieux professionnels fort variés, au premier rang desquels figurent personnalités politiques et administratives, de Jean Foyer à Philippe Séguin, et représentants des métiers de la culture et du patrimoine. Avec la remise d'un rapport au ministre en février, l'année 2007 et ses échéances présidentielles chargées de promesses, peut certainement marqué à la fois le point d'orgue de la mobilisation et le début d'un certain reflux, peut-être provisoire. La disparition successive de plusieurs porte-voix du comité a sans aucun doute contribué à cet essoufflement : après Maurice Druon, Alain Boumier disparaissait fin 2009, avant Philippe Séguin en 2010 ou enfin Jean Dutourd au début de l'année 2011.

III. 2 : Page d'accueil du site du comité national pour la reconstruction des Tuileries (fermé)

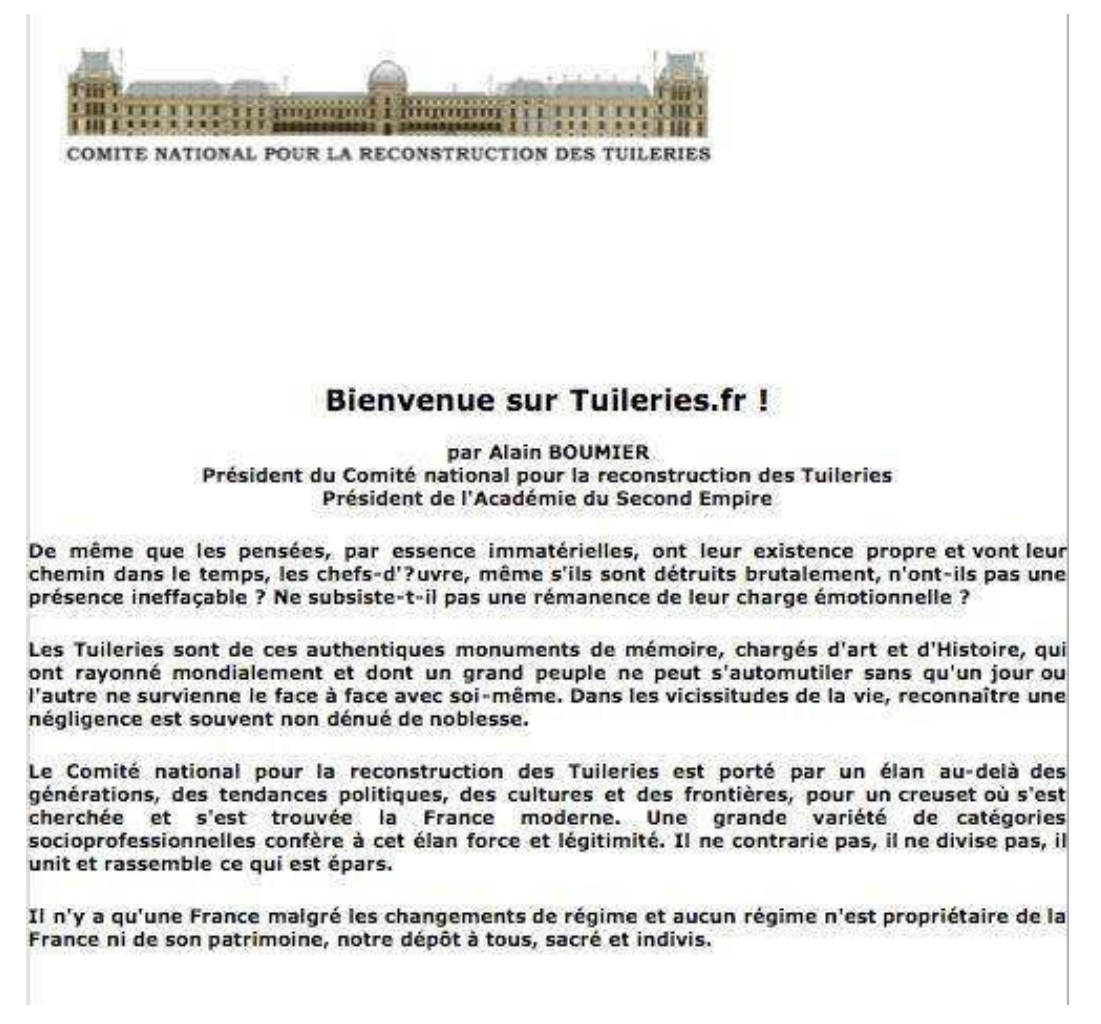

Capturée en novembre 2007 
5 L'intervention orale ${ }^{4}$ qui commande l'écriture du présent article, s'inscrivait, en novembre 2007, dans ce moment suspendu à une autorisation étatique qui n'est finalement pas venue. Quelque mois après l'élection d'un nouveau président de la République, aucun relais n'a finalement vu véritablement le jour. Le caractère inabouti et incertain de cette mobilisation en devenir a commandé un ajustement du regard sur ce qui depuis lors reste une émotion patrimoniale potentielle, car toujours disponible. D’autant que la « rémanence » supposée du lieu semble charger le vide qui lui fait place d'une fatalité irréversible.

6 Pourquoi en effet l'émotion patrimoniale proprement dite, en tant que conjonction d'un mouvement d'opinion ayant le pouvoir de déborder la sphère professionnelle des métiers du Patrimoine, n'a pu vraiment se déployer? Comment, à l'inverse, le projet de reconstruction a pu être la source (et le symptôme) d'un débat émotionnel au sein de ce même milieu du Patrimoine? La spécificité - architecturale et politique - du lieu de mémoire et d'anti-mémoire que forment les Tuileries est-elle vraiment au cœur de l'argumentaire déployé par le comité?

7 Toutes ces questions exigeraient une étude en profondeur des attendus et des moyens mis en œuvre par une mobilisation patrimoniale finalement restée lettre morte. À défaut d'une analyse " ethnographique » de ce qui pourrait être appréhendé, peut-être, comme une émotion patrimoniale contemporaine, cet article se propose d'évaluer la pertinence et les implications de la "rémanence » topographique et émotionnelle supposée du Palais. Pour ce faire, il s'agit de revenir, moins sur l'histoire du palais lui-même, qui a récemment fait l'objet d'une belle monographie ${ }^{5}$, que sur celle des motifs de sa destruction, longue et complexe, de mai 1871 à janvier 1883, de l'incendie du bâtiment par la Commune agonisante à son démontage effectif par la République naissante. La mise en œuvre, entre 1884 et 1888, au lendemain de la destruction, d'un projet de reconstruction d'un nouveau palais, dédié à un "musée de la Révolution française », en vue notamment du centenaire de la Révolution, sinon de la République, donne la mesure $\mathrm{du}$ refoulement autant que de la rémanence dont le lieu a pu faire l'objet. L'avortement récurrent de cette émotion " reconstructiviste ", qui, en fait, s'égrène tout au long du XX siècle, par une série de projets tout aussi inaboutis permet de saisir l'écheveau qui commande une articulation singulière où patrimoine et politique s'incarnent mutuellement l'un par l'autre et l'un en l'autre.

\section{Les ruines des Tuileries : une histoire « suspendue » qui reste à écrire}

8 L'histoire de la «mort lente des Tuileries » durant la décennie 1870 est fort bien documentée par les archives de la direction des bâtiments civils et des palais nationaux conservées au sein de la sous-série $\mathrm{F}^{21}$ des Archives nationales ${ }^{6}$. Cet ensemble archivistique, qui émane essentiellement du second bureau (ayant parfois rang, selon les moments de réforme administrative, de division), dit " des Palais nationaux ", offre une belle suite de dossiers, enregistrés annuellement, mais en partie digérés au fur et à mesure du suivi des affaires par la continuité du travail administratif ${ }^{7}$.

9 La conservation des dossiers permet d'accéder aux projets localisés sur le périmètre du palais au cours de cette période. Ainsi que c'est la règle au sein des archives administratives du XIX ${ }^{\mathrm{e}}$ siècle, les dossiers contiennent la minute des lettres expédiées 
par le bureau, quelques rares notes internes, fort difficiles à interpréter, mais surtout la documentation reçue, sans grande hiérarchisation entre les projets et correspondance de l'Agence officielle du Palais, dirigée par Lefuel jusqu'en $1880^{\circ}$, et les projets ou avis spontanés émanant de la société civile. Ces documents d'archives "projectifs " et entremêlés, dans une confusion que le dépouillement systématique ne permet pas toujours de résoudre, apparaissent comme les symétriques prospectifs des ruines encore présentes : le palais des Tuileries, «ni tout à fait détruit, ni encore reconstruit » pendant cette longue latence de onze années, apparaît comme travaillé par deux temporalités contradictoires, relique d'un Empire tout juste anéanti dont la République balbutiante n'ose encore décider du sort définitif. Pour apprécier toute la puissance et toute l'ambiguïté de ces ruines, il faut en effet aujourd'hui prendre la mesure de la "véhémence topographique " qu'a pu représenter pendant ces onze années ce gigantesque palais incendié mais non encore abattu, en plein centre de Paris, au cœur même du grand récit architectural et national matérialisé par le périmètre LouvreTuileries. L'histoire de cette latence, de ce long refroidissement des ruines des Tuileries, au cours duquel l'État semble en partie en retrait, dans une position attentiste vis-à-vis des projets qui lui sont soumis, mériterait d'être racontée dans le détail pour ce qu'elle révèle des atermoiements d'une République qui peine à accoucher douloureusement d'elle-même.

10 Au-delà de l'écho troublant que l'on peut percevoir entre le destin hésitant du régime politique au cours de la décennie 1870 et la confusion qui entoure le sort du palais des Tuileries pendant la même période, il s'agit en parallèle de souligner les enjeux méthodologiques induits par le dépouillement de ces boites d'archives de la série $\mathrm{F}^{21}$ couvrant les années 1871-1891: que révèlent ces boites, gorgées de projets par définition avortés, inaboutis? Qu'expriment ces idées, ces requêtes, ces esquisses dessinées ou coloriées ? Que disent-elles de l'histoire politique et culturelle de la France de ces années 1870 ? Disent-elles seulement « quelque chose »? Ne faudrait-il pas, plutôt que de risquer de s'y perdre, laisser ces esquisses sans issue là où elles sont, aux oubliettes de l'historiographie? Ne vaudrait-il pas mieux concentrer l'attention sur les projets finalement réalisés, en tant qu'ils sont les jalons d'une histoire effective, par opposition aux flâneries hasardeuses d'une histoire projective?

11 C'est précisément l'accumulation de ces nombreux projets, dans leur très grande diversité et leur inaboutissement, qui nous parait révéler la force d'attraction, ou mieux, "d'aimantation " du lieu, et ce du fait même du sentiment d'absence et de manque crée par le spectacle des ruines, dont les contemporains comprennent sans doute qu'elles annoncent - à plus ou moins brève échéance - le vide (ill.3 et 4). Nous pouvons suivre ici les propositions d'Éric Fournier qui n'hésite pas à parler d'une "éloquence du cadavre ", pour souligner toute la force qui se dégage paradoxalement de ces «charognes minérales $»^{10}$ : la ruine, le spectacle de la ruine, annonce sa disparition prochaine, et même si cette annonce " traîne en longueur ", elle appelle donc à combler le vide à venir par des projets, des projections, des esquisses, des dessins et des desseins qui permettent d'imaginer un lendemain et un au-delà des ruines. Plus largement, la curieuse conservation, au sein des archives des Bâtiments civils, de projets spontanés et sans échos, consacrés à l'occupation de l'espace vacant Tuileries nous semblent dessiner les contours incertains d'une histoire qui, sans céder aux tentations de l'uchronie ou de l'histoire "contrefactuelle», donnerait toute sa place aux «champs des possibles » de chaque séquence historique; une histoire parafactuelle, en quelque sorte, une histoire 
potentielle ou suspendue, indispensable en particulier pour saisir la vérité des périodes marquées, précisément, du sceau du doute et de l'incertitude. Cette histoire serait résolument contemporaine ou plus précisément simultanée en tant qu'elle refuse de taire les «possibles contemporains» inhérents à chaque époque. Cette proposition méthodologique pourrait alimenter une histoire totale du lieu, possiblement « rémanent » des Tuileries, dont le déploiement excèderait largement le questionnaire et le format du présent propos. En se concentrant sur les semaines et mois qui suivent l'incendie, il est possible de restituer les contradictions entre une histoire potentielle de la reconstruction et les fatalités d'une destruction, très vite inéluctable.

\section{3 : Proposition de reconstruction d'un double palais pour le ministère de l'Intérieur et la Présidence de la République}

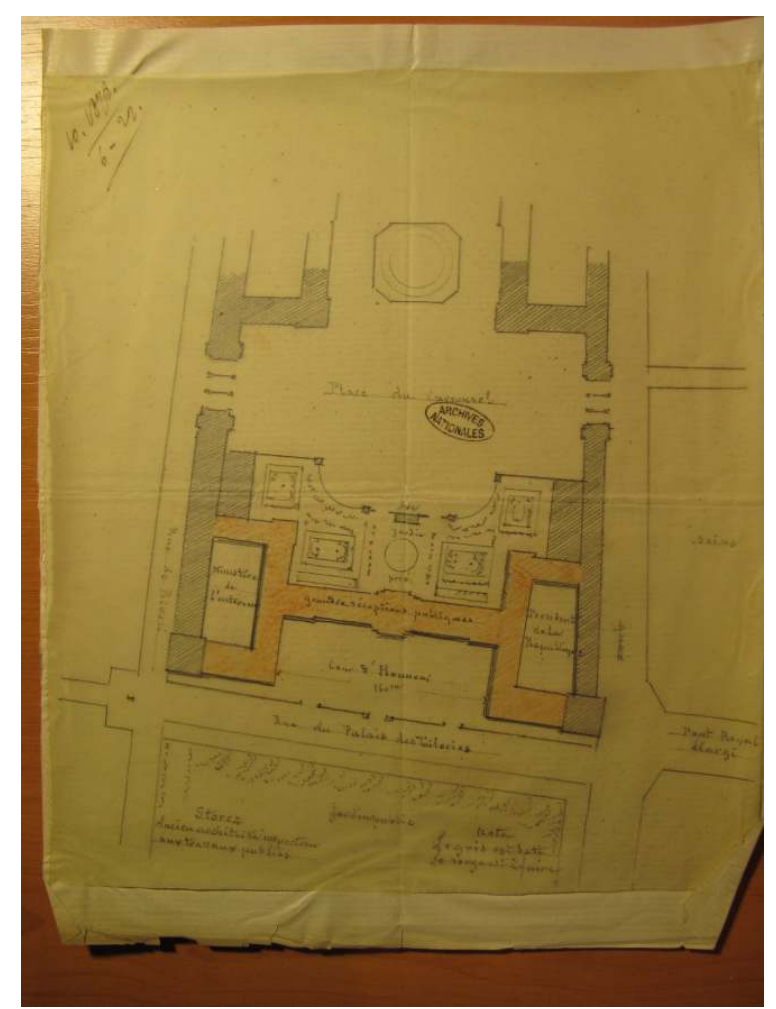

Par Storez, ancien inspecteur des travaux publics, soumis à la direction des bâtiments civils, 12 juin 1876 : Arch. nat. $F^{21} 881$, « dossier 8 » 
III. 4 : Proposition d'aménagement de l'espace du Carrousel

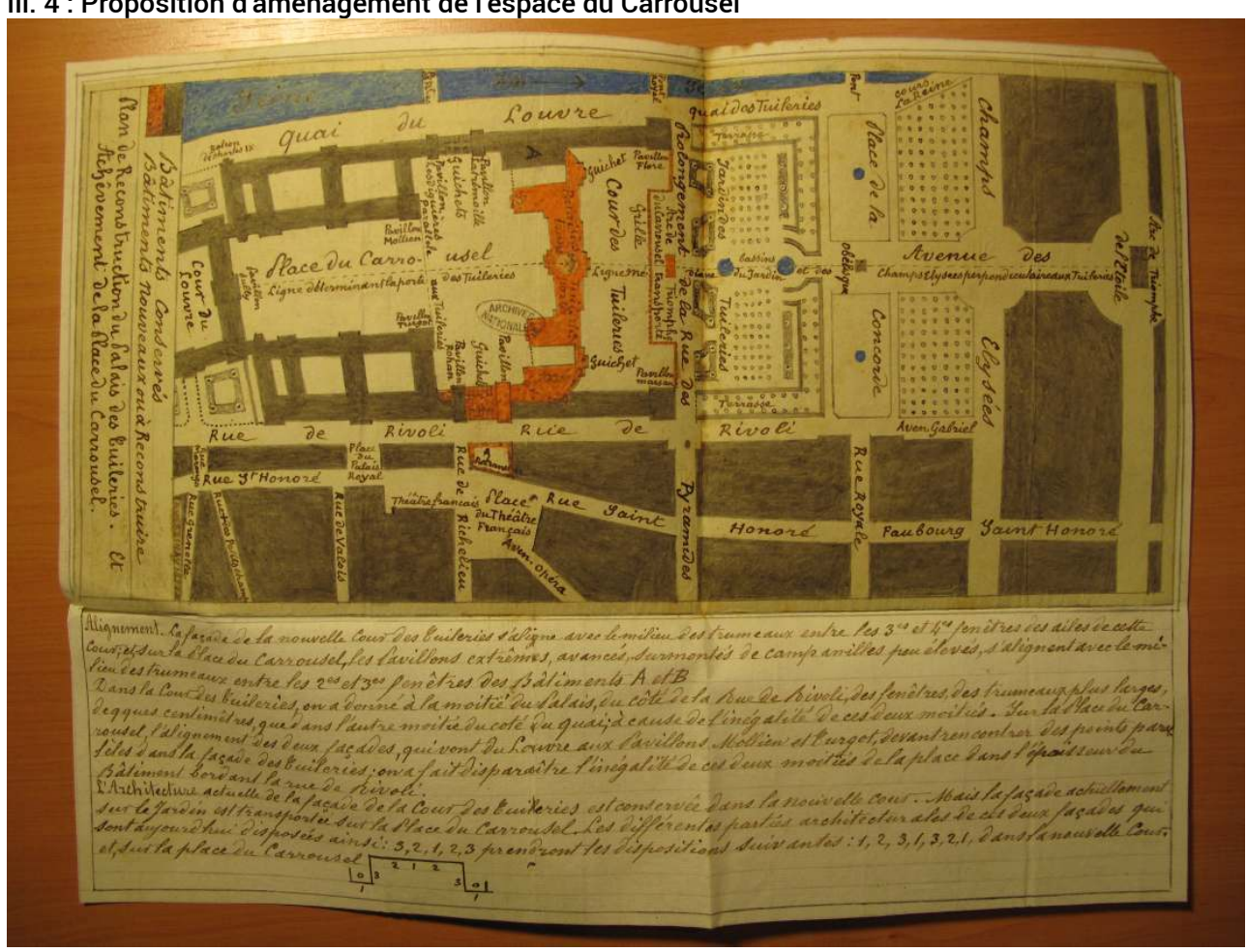

Par A. Verzinay, soumis à la direction des bâtiments civils, novembre 1877 : Arch. nat., F' 881 , dossier 8

\section{1-1873 : un destin architectural précocement scellé ?}

12 Dès juin 1871, quelques jours après l'incendie du 23 mai, l'ambiguïté fondamentale des ruines du palais des Tuileries apparaît déjà au grand jour: alors que certains des pourfendeurs de la Commune souhaitent conserver les ruines en l'état pour désigner aux yeux de tous les « crimes » de ce régime politique éphémère, d'autres considèrent que la démolition est la condition nécessaire d'une possible future reconstruction, à l'identique ou non. Dans le rapport qu'il rédige le 4 juillet 1871 au nom de la commission parlementaire « ayant pour objet la conservation des ruines des anciennes Tuileries », le député Henri Margaine met en avant pour sa part la dimension «scientifique » de ces reliefs palatiaux: "Si dans le centre de Paris nous maintenons à l'état de ruines des monuments d'un autre âge, c'est pour répondre à un besoin de la science, c'est un souvenir archéologique que nous conservons. C'est une affaire d'architectures bien plus qu'une affaire de sentiments $»^{11}$. Il répondait en cela au député de Lozère Théophile Roussel qui avait défendu quelques jours plus tôt une conservation explicitement expiatoire : «Il peut être utile de laisser subsister ces ruines pour stigmatiser aux yeux de ceux qui viendront après nous les auteurs des ces épouvantables calamités ${ }^{12}$. La formulation du député Roussel est frappante, parce qu'elle suppose que les ruines des Tuileries pourraient rester en place pendant plusieurs décennies, destinées justement à une fonction pédagogique pour les générations à venir, et non pas seulement à une expiation ponctuelle destinée à ne pas durer au-delà d'une période post-traumatique bornée dans le temps. 
13 Hector Lefuel, architecte en charge du Louvre et du palais des Tuileries depuis 1855, se situe dans la même logique qu'Henri Margaine. Dès le premier rapport qu'il rédige, dix jours seulement après l'incendie, le 5 juin 1871, il met en avant lui aussi la dimension " archéologique " des fouilles entamées dès le refroidissement du brasier: "Mon attention s'est tout d'abord portée [...] sur la salle de stuc [...] où l'on disait qu'avaient été rapportées du Garde-Meuble de la Couronne des statuettes et des objets d'art provenant de l'Hôtel de Mr. Thiers. Dès samedi matin j'ai fait commencer le déblaiement sur ce point; il a été continué hier toute la journée; il se poursuit encore, mais malheureusement sans aucun résultat fructueux ${ }^{13}$. Dans l'immédiat, la conservation et la protection des ruines vise donc simplement et pragmatiquement à tenter de retrouver les objets de valeurs qui sont dispersés au milieu des décombres, dans une démarche résolument archéologique. Pour assurer la sécurité des fouilles, Hector Lefuel bénéficie du soutien militaire: "Quelques sous-officiers du Génie [...] sont constamment avec les ouvriers ; ils les dirigent [...] aussi bien dans leurs recherches et l'enlèvement que dans le triage que j'ai fait faire des différents objets que rencontre la pioche des travailleurs [...]. Très habilement secondé par l'autorité militaire [...] je crois pouvoir vous assurer M. le Directeur, qu'aucune soustraction ne sera possible ». La sauvegarde provisoire des ruines du Palais vise donc moins à garantir une sauvegarde ultérieure du contenant - le bâtiment - qu'à assurer la sauvegarde immédiate de son contenu. Cette interprétation est confirmée par la suite du rapport de Lefuel, qui se montre très pessimiste quant à une possible restauration future: "plus je m'avance au milieu de ces ruines toujours fumantes et plus je vois combien il est difficile d'apprécier encore l'étendue de nos désastres ». Plus fondamentalement, Lefuel fait la différence entre les ailes latérales du Palais, dont la qualité de construction laisse espérer une possible restauration, et le corps du bâtiment central, dont la piètre qualité architecturale originelle laisse déjà entrevoir une démolition inévitable. Sa description est fort précise et de ce fait particulièrement cruelle pour les partisans actuels de la reconstruction du bâtiment central des Tuileries :

«Quant au corps de Bâtiment situé entre le Pavillon de Flore et le Pavillon de Marsan, en y comprenant ce dernier, c'est à dire quant au vieux Palais des Tuileries, je considère que vu la condition essentiellement défectueuse de sa construction originelle et les modifications si nombreuses et si peu réfléchies qu'on lui a fait subir, il ne saurait se prêter à aucune restauration sérieuse. Je ne pense pas que les découvertes que je puis avoir à faire modifient mon sentiment présent ; je crois le Palais perdu et j'abandonne la poursuite du projet de sa consolidation ».

14 La conception architecturale médiocre du bâtiment originel, aux dires mêmes de Lefuel, justifie donc par avance sa future démolition. Lefuel ne porte pas le même jugement sur "Les Tuileries nouvelles, c'est à dire l'aile en retour sur le Quai", pour lesquelles il souligne que "les dégradations du bâtiment en tant que grosse construction sont relativement minimes, elles sont du moins aisément réparables »; de même pour l'aile nord du Palais situé « depuis le Pavillon de Marsan jusqu'au Guichet de l'Échelle », dont l'architecte souligne que «les conditions de construction infiniment meilleures me permettraient d'espérer qu'une restauration de cette importante partie serait très possible ». Hector Lefuel, qui a pourtant dirigé les travaux d'achèvement du "grand dessein » depuis 1855, porte donc dès le 5 juin 1871 un jugement sans appel sur la partie centrale du Palais dont la démolition sera finalement décidée onze ans plus tard, en juin 1882.

15 L'impression laissée par l'analyse du tout premier rapport de Lefuel se retrouve pendant presque toute la décennie, et ce dernier se borne à reprendre les mêmes arguments : en 
réalité, dans l'esprit des responsables comme dans celui des observateurs et des entrepreneurs liés au dossier, la démolition de la partie centrale du palais des Tuileries semble bien, à terme, inéluctable; tout se passe comme si chacun savait que la démolition aurait lieu dans un avenir plus ou moins proche, tout en sachant que cette démolition ne pouvait se faire dans l'immédiat, tant les cendres du brasier étaient encore trop chaudes, au propre comme au figuré. Dans cette attente, on commence par traiter les ruines du palais comme un stock de matériaux destiné à réparer les parties endommagées du Louvre; ainsi le 8 novembre 1871 autorisation est donnée de « réemployer les plombs et les fers trouvés dans les décombres pour les travaux à exécuter d'urgence au Musée du Louvre et à l'aile neuve des Tuileries $»^{14}$. La logique est évidente : on démembre les Tuileries pour sauvegarder le Louvre, ce qui montre bien la différence de statut entre les parties que l'on sait promises à la démolition et celles qui seront sans aucun doute restaurées. Les dossiers du bureau des palais nationaux consacrés au Palais des Tuileries évoquent des déblaiements, des démolitions, des enlèvements de fragments, et aucune pièce n'évoque des travaux de consolidation, de renforcement des fondations ou de remise en place d'une couverture même provisoire, ce qui est pourtant la première des mesures à prendre pour empêcher qu'un bâtiment incendié ne se dégrade à brève échéance. Le 11 juillet 1871 un entrepreneur « demande la permission de fouiller dans les gravats et poussières venant des Tuileries qui se déchargent à Sèvres "; le 8 août suivant on approuve les devis «relatifs aux déblaiements des parties incendiées»; le 29 novembre de la même année on procède à la vente d'objets divers et de matériaux provenant de la démolition " [sic]. Dans les mois qui suivent, à la demande d'Adolphe Thiers, Lefuel se voit dans l'obligation de fournir divers scénarii possibles, qui alimentent un projet de loi global sur le financement de l'ensemble des reconstructions des bâtiments incendiés par la Commune (ill.5), et dont les archives du bureau des palais nationaux ne conservent qu'une trace partielle ${ }^{15}$. 
III. 5 : Ministère des travaux publics, direction des bâtiments civils. Réparation des dégâts causés à divers monuments et édifices par le bombardement et par l'insurrection. Estimation des dépenses.

\section{Second semestre 1871}

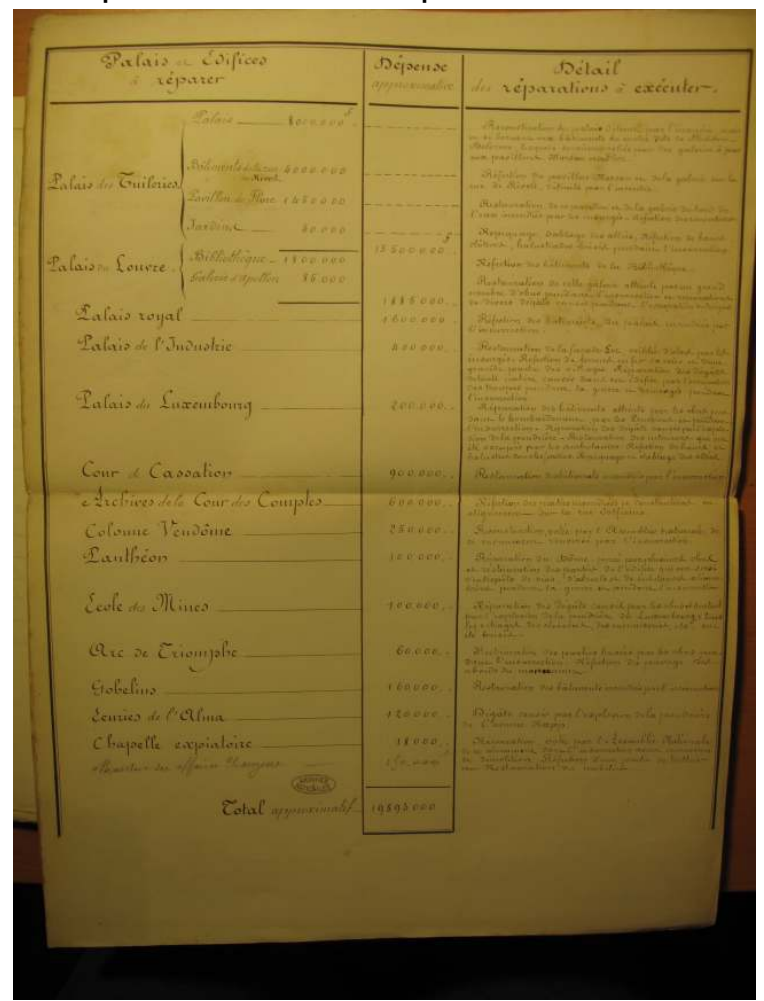

Arch. nat., $F^{21} 881$, dossier 2

Au cours de ce moment suspendu et néanmoins fatal, où l'eau de pluie relaie sans cesse au quotidien ce que le feu d'une nuit n'a pu atteindre, le tournant décisif quant au devenir du Palais est en fait fort précoce : dès avant la chute de Thiers en mai 1873, Lefuel obtient ce qu'il demande depuis son premier rapport, soit l'ablation, pour des raisons de sécurité, des galeries latérales reliant le noyau du "vieux Palais » aux deux pavillons de Flore et de Marsan. Le cordon ombilical entre le Palais de Catherine de Médicis et le Louvre des rois est ainsi rompu par le même main qui en avait assuré six ans plus tôt la clôture, accomplissant l'achèvement décidément éphémère du «Grand dessein » en 1867. Ainsi isolé de ses deux lourdes galeries, qui depuis un texte resté célèbre de Chateaubriand en 1831, semblaient concentrer toutes les critiques esthétiques et architecturales, le vieux palais en ruines est désormais condamné. Lefuel concentre en effet dès lors toute son énergie et ses chantiers à restaurer et reconstruire les deux ailes et pavillons qui sont destinés à «terminer» le Louvre. En menant à bien le chantier d'aménagement des façades sud et nord des pavillons de Marsan et de Flore, Lefuel précipite, à partir de 1874, le sort des Tuileries, désormais réduit à un Pavillon de l'Horloge, central certes, mais fort étroit, et bientôt irrémédiablement isolé au sein d'une vaste cour du Carroussel qui ne demande qu'à rejoindre l'espace du grand jardin des Tuileries. La présence des ruines est ainsi désormais inorganique, et bientôt perçue comme un obstacle au cœur d'une vaste trouée urbaine, possiblement jardinée.

17 Le point de non retour semble avoir été ici franchi: sous prétexte d'une épuration stylistique, sinon patrimoniale, du palais, les choix de Lefuel n'entraînent pas seulement une perte décisive d'intégrité physique, mais provoque plutôt une disqualification volumétrique irrémédiable : à l'issue de cet arbitrage silencieux et qui n'a pas fait l'objet 
d'un débat législatif, le périmètre et le volume même de ce que l'on peut, fonctionnellement, appeler un " palais » se trouvent très largement abolis.

C'est donc au lendemain de ce "passage à l'acte », dont la responsabilité repose toute entière sur Lefuel, à partir de la fin de l'année 1873, alors que la démolition semble imminente pour tous, que l'opinion publique, avant les députés et les sénateurs, s'émeut véritablement et que les arguments strictement patrimoniaux se déploient dans l'espace public : un bel indice archivistique le suggère, dans la mesure où le plus ancien article de presse collationné et conservé dans les dossiers afférents aux Tuileries date de la fin de l'année $1874^{16}$. C'est aussi à la veille de cette décision fatale que, pour la première fois semble-t-il depuis l'incendie, l'administration patrimoniale proprement dite, sous la tutelle du ministère de l'Instruction publique, semble se sentir concernée par une prise en charge des restes du palais: ainsi le 15 mars 1873 «La commission des Monuments historiques a pensé qu'avant l'accomplissement de l'œuvre de démolition il serait intéressant de faire relever sur place les fragments les plus beaux de cette ancienne résidence. Ce travail lui paraît d'autant plus désirable que l'historique de la construction des Tuileries est entouré d'incertitudes et que l'état actuel des bâtiments qui sont à l'intérieur débarrassés de leurs enduits peut, en facilitant les recherches, fournir des indications précieuses $»^{17}$. Le palais des Tuileries apparaît ici pour ce qu'il devait être effectivement dans l'esprit des contemporains : un écorché architectural à ciel ouvert, dont la disparition imminente justifie une ultime autopsie. Le 29 octobre 1873 un dossier documente la « demande de deux fragments de sculptures provenant de la démolition des Tuileries pour le Musée royal d'archéologie de Lisbonne ». Dans sa lettre, le chevalier da Silva parle précisément d'un édifice "presque démoli", dont il conviendrait de sauvegarder un fragment " comme spécimens du style de l'architecture » ${ }^{18}$. Carrière à ciel ouvert, stock de matériaux, parc archéologique: quels que soient les documents consultés, on voit que les ruines des Tuileries sont en somme déjà condamnées en pensée, sinon en actes.

19 Au cours des deux premières années qui suivent l'incendie, si le bâtiment du palais semble promis à un proche abandon, la vie reprend rapidement son cours dans le jardin des Tuileries, comme en témoignent les très nombreuses demandes qui sont faites au cours du printemps et de l'été 1872 : « vente de coco ", «vente de petits gâteaux », "demande d'autorisation de faire circuler une petite voiture attelée de chèvres", "demande d'autorisation d'établir dans le jardin des Tuileries un petit théâtre dit Guignol », remise en adjudication de la location de chaises... Ces demandes ne sont pas toujours acceptées mais elles témoignent en tous cas clairement de la réouverture du jardin au public, dès le printemps suivant l'incendie. D'autres demandes montrent que certains se projettent déjà dans un futur qui fait abstraction en quelque sorte de la présence physique ou du moins fonctionnelle du palais : le 9 novembre 1871 par exemple le comte de Brunet de Puisaye dépose une «demande de concession d'un emplacement de 14 à 16 mètres de diamètre pour y élever un pavillon de fer et de verre [...] pour y établir un Diorama universel des merveilles de la nature et de l'art »; l'année suivante, «Mr Tissandier demande à établir un ballon captif dans la cour ou dans le jardin des Tuileries "... tout se passe comme si le palais, dont la fonction est annulée de facto par l'incendie, se retrouvait cerné et comme absorbé par la rapide appropriation publique des jardins qui le bordent immédiatement. La présence du public aux abords immédiat du palais incendié doit être également reliée à la question des voies de circulation, qui concourt elle aussi à accentuer l'étranglement topographique de l'ancien palais. En effet, de nombreux documents témoignent de la 
volonté des riverains de la rue de Rivoli de pouvoir traverser plus directement le quadrilatère Louvre-Tuileries lorsqu'ils doivent se rendre sur la rive gauche, sans s'obliger à un long détour soit par la place de la Concorde soit par les guichets du Louvre. Un épais dossier documente ainsi un projet de tunnel permettant la « traversée du Jardin des Tuileries, soumis à S. M. l'Empereur par Léon Lesueur (typographe) en 1856, 1860, 1867 et 1870 . Adressé à $\mathrm{M}$. Thiers et à ses Ministres en décembre $1871 »^{19}$. Plan à l'appui, Léon Lesueur joint à sa demande une pétition signée par pas moins de 177 riverains et martèle son argument principal: «de la rue de Bellechasse à la rue de Castiglione la distance actuelle, pour les personnes munies d'un paquet quelconque, est de 1750 mètres ; par le Tunnel elle ne serait plus que de 487 mètres ». Or, tout indique que l'incendie du palais a pratiquement réglé le problème, en permettant la libre circulation du public aux abords immédiat du palais, entre la rue de Rivoli et le Pont Royal, évitant ainsi aux piétons un très long détour. Dans les documents figurés des mois qui suivent l'incendie, l'allée qui borde à l'ouest le palais incendié est bientôt appelée « rue des Tuileries » : effet induit de l'incendie, cette nouvelle voie de circulation apparaît comme un acquis de la circulation urbaine dès l'automne 1871, qui serait à l'évidence remis en cause par la restauration du palais, puisque sa façade ne pourrait être immédiatement bordée par une rue.

L'analyse des deux premières années qui suivent l'incendie dans une perspective large d'histoire urbaine (destination effective des ruines du palais, usages des jardins, logiques de circulation) permet de relativiser considérablement les débats mieux connus qui se développeront dans les années suivantes à propos des projets de restauration ou de reconstruction des Tuileries. Le palais, écorché vif par l'incendie, est en effet rapidement absorbé par le jardin qui le borde et sa terrasse en façade est bientôt remplacée par une voie de circulation dont la nécessité est reconnue depuis longtemps : en réalité, son sort paraît scellé de facto, bien avant que ne soient officiellement tranchés les débats ultérieurs mené sur la scène politique complexe des années 1870.

\section{La disparition des Tuileries ou la rupture émotionnelle et symbolique et Patrimoine et Politique}

21 L'histoire de la destruction inéluctable des Tuileries n'a pas, depuis un siècle, suscité un grand engouement historiographique. Après les études fondatrices d'Emmanuel Jacquemin, qui avait pu consacrer au moins deux ouvrages illustrés au Palais ${ }^{20}$, Guillaume Fonkenell a tout récemment analysé en profondeur son histoire architecturale, à l'issue d'un travail d'investigation d'archives anciennes en grande partie inédites. De ce fait, l'histoire, et, a fortiori, l'avenir rompu des projets de reconstruction ne font pas partie de son objet d'étude. Dans son dernier chapitre, «La fin des Tuileries $»^{21}$, il propose toutefois une synthèse dialectique des valses hésitations administratives et législatives qui conduisent, en une décennie, de juin 1871 à juin 1882, de la certitude d'une sauvegarde, au moins partielle, du gros œuvre de Philibert de l'Orme à la dynamique irréversible du démantèlement et de la démolition complète. Cette synthèse fait écho à un bouquet de publications récentes dues au chercheur américain Louis Iandoli, qui à partir d'un point de vue plus extérieur, s'est attaché à comprendre le paradoxe qui renverse en une décennie le rapport de forces autour de la reconstruction ${ }^{22}$. Dans les deux cas, l'argument politique, sinon même constitutionnel est décisif : et l'histoire de la destruction semble suivre pas à pas la lente conquête de la majorité parlementaire par les républicains. À 
partir de la convocation d'une partie des archives des Bâtiments civils, ces publications se proposent de dresser une synthèse au sein de laquelle l'expertise professionnelle des architectes en charge du bâtiment en ruines semble balayée sans cesse par une sorte de fatalité politique. L'autorité de Lefuel, avant celle de Garnier, qui lui succède après sa mort à la fin de l'année 1880 - mais pour la seule partie en ruine (ce qui ne fait que renforcer le scellement du sort du « palais » initié en 1873) - semble sans cesse mise à mal par la confusion et l'incertitude des rapports de forces politiques au sein des deux chambres, notamment après les élections législatives de 1876, et avant la victoire républicaine au Sénat de 1879. La succession des commissions d'experts, considérée comme le fruit d'une " machine administrative kafkaïenne $»^{23}$, mobilise pourtant, à partir de 1876, les grands acteurs de la politique de restauration des monuments historiques, jusqu'à Viollet-le-Duc lui-même. S'il reste alors la figure de proue, sinon la statue du commandeur, de l'élite architecturale des Monuments historiques, ce dernier se révèle, selon Fonkenell et Iandoli, tout aussi impuissant pour interrompre la marche inéluctable à la destruction. D'autant que l'inertie du temps qui passe, qui désagrège au quotidien les ruines, demeure la meilleure alliée de ceux qui souhaiteraient les voir disparaître. Ainsi, à la lecture de ces études récentes, est-on tenté de supposer une forme de manipulation, sinon de complot, notamment au moment du décisif passage du fidèle collaborateur de Gambetta, Charles de Freycinet, au ministère des Travaux publics, entre décembre 1877 et décembre $1879^{24}$, visant à instrumentaliser l'enlisement des projets par leur accumulation. Il est vrai que l'état anarchiquement «archivé » de ces derniers dans la série $\mathrm{F}^{21}$, de par le démantèlement et les lacunes manifestes au sein des deux principaux dossiers afférents à la « reconstruction » du palais ${ }^{25}$, puis à « l'enlèvement » des ruines ${ }^{26}$, laisse une impression de grande confusion. Les reclassements et les insertions opérés successivement au sein de ce qui fut un moment des dossiers courants ouverts, laissent pour finir un état « définitif » bien difficile à interpréter, y compris jusqu’à la chronologie et l'enchevêtrement des commissions et des débats. Tel un tonneau de fragments déconnectés, ces deux dossiers se complètent paradoxalement, davantage qu'il s'excluent, le second comprenant des pièces décisives pour la compréhension, de ce fait posssiblement perceptible comme « kafkaïenne », des logiques de blocage et de déblocage successifs du processus administratifs et du débat législatif. Au total, il est difficile de démêler l'écheveau fabriqué par le désordre archivistique contemporain à la production des dossiers, de la confusion administrative des procédures, voire de la manipulation des manœuvres politiques supposées.

Sans doute la chute du gouvernement de Thiers, le 24 mai 1873 (soit deux ans jour pour jour, après l'incendie du palais) interrompt définitivement le premier processus de restauration des ruines, initié à l'automne 1871, une opération que Lefuel avait estimée, avec un enthousiasme très modéré, comme pouvant être menée au prix d'un investissement de 8,4 millions de francs. Cette chute est surtout le point de départ d'une séparation structurelle entre le patrimoine et la politique. Le projet de loi afférent, préparé durant l'hiver 1871-1872 $2^{27}$, butait en effet sur un problème qui constitue le point d'achoppement de tous les projets ultérieurs : il ne s'agit pas seulement de décider de la forme architecturale légitime des Tuileries restaurées ou reconstruites, mais bien de savoir ce que pourrait être la fonction même du palais. En somme, en désolidarisant le contenu projeté du palais de la valeur architecturale de son contenant subsistant, il est bien difficile d'y voir clair. 

scientifique, ou par «amour» de l'histoire de l'art, d'une architecture supposée référentielle de la Renaissance, bien que mise à mal par trois cents ans d'aménagements successifs, n'a jamais permis d'emporter l'adhésion, en dehors des milieux historicistes des architectes, et ce, pourrait-on dire, dès l'été 1871. Tant que Thiers est au pouvoir, l'ambiguïté de sa personne - entre ancien ministre de la monarchie de Juillet, fantôme du bonapartisme, et apôtre inattendu d'une République plus qu'incertaine - permettait d'envisager la possibilité de sauver le palais par le prolongement de sa fonction résidentielle suprême. Depuis le 6 octobre 1789, et l'installation forcée du roi à Paris, hormis au moment du Directoire et durant la brève parenthèse élyséenne de 1848 à 1851, les Tuileries ne sont pas seulement, par tradition ou inertie, le siège du pouvoir exécutif : l'installation dans le Palais, sinon sa " prise », en cas de changement de régime, constitue, de fait, un geste de légitimation en soi. Son occupation signifie la prise du pouvoir, au point de constituer l'équivalent d'une immense salle du trône, de vaste lieu d'onction et d'institution : l'équivalent d'une regalia architecturale. nécessaire lieu de mémoire partagé entre tous les régimes successifs de la France depuis la Révolution. Non content d'être non tout à fait réductible à une quelconque raison monarchique ou impériale, les Tuileries ont de plus la particularité d'être un véritable sanctuaire de l'histoire du pouvoir législatif : la salle de la Convention, aménagée au nord du palais, fut la première salle d'assemblée spécifiquement aménagée pour cette fonction en France... Certes les aménagements consulaires et impériaux du Premier Empire avaient gommé toute trace de ce moment d'un premier monument du législatif.

En parallèle néanmoins, subsistait dans l'environnement immédiat même du palais un héritage de la Révolution qui n'avait pas été effacé, mais bien au contraire approfondi par chaque régime successif : il consistait précisément en l'association entre le palais de la Nation et celui de la Science et des Arts dans un même ensemble Louvre-Tuileries. On peut même dire que cette cohabitation entre souveraineté politique et souveraineté des arts, a été renforcée pendant le Second Empire. Pour toutes ces raisons, l'assimilation, jamais vraiment énoncée en ces termes dans l'historiographie récente, mais toujours sous-entendue, entre défenseurs de la restauration/reconstruction et promoteurs de la monarchie d'une part, et acteurs de la destruction/disparition et partisans de la République d'autre part, offre une grille de lecture insuffisante, pour ne pas dire réductrice. La complexe dialectique qui guide et commande l'enchevêtrement des positions administratives, politiques et patrimoniales, qui mène irrémédiablement à la destruction du palais ancien, ne repose donc pas sur une opposition binaire, entre monarchie et République, ou pour le dire autrement, entre la Droite et la Gauche, mais bien sur une équation à quatre inconnues identifiables.

L'inventaire, même partiel, des multiples argumentaires et projets de reconstruction, montre en effet une série kaléidoscopique de positions, dispersées dans l'espace politique. Dès le lendemain de l'incendie des républicains convaincus, voire radicaux, sont ainsi pleinement favorables à une reconstruction à destination politique. Ainsi dès 1872, et plus encore à partir du retour des chambres à Paris en 1879, il semble évident aux yeux de certains républicains que la charge symbolique ultime consisterait à voir le palais des Tuileries, en écho à sa fonction sous la Convention, transformé en siège de deux chambres. Sadi Carnot, le petit-fils de l'artisan des victoires de l'an II, ne tente-t-il pas, au cours de l'été 1879 , de susciter un débat sur l'installation des deux chambres aux Tuileries 
${ }^{28}$ ? La démolition, alors à l'ordre du jour n'est pourtant pas encore votée. Joseph Reinach, près de 10 ans après la victoire du vide entre Carrousel et Tuileries, tentera une ultime fois sans doute de remettre ce projet à l'ordre du jour ${ }^{29}$.

Inversement, et ce dès 1876 , le vicomte d'Aboville, qui fut un des plus vifs défenseurs d'une restauration totale ainsi qu'un monarchiste déclaré, préférait mettre en avant l'idée d'une reconstruction à fonction muséale, en proposant l'installation, inattendue, quoique évoquée depuis plusieurs années par les partisans d'une conservation des reliques de Philibert de l'Orme, d'un musée pour les arts modernes et contemporains ${ }^{30}$. Par-delà la démolition votée en janvier 1882, sous l'éphémère ministère des arts d'Antonin Proust, ainsi qu'ont pu le relever avec malice et raison tous les historiens de la disparition des Tuileries, il se trouve enfin que le seul projet ayant atteint les cimaises du débat parlementaire et du projet de loi fut bien celui d'un «musée de la Révolution française », en lieu et place des Tuileries, entre 1884 et 1888 (ill.6) ${ }^{31}$. L'ampleur du dossier conservé dans les archives du bureau des palais nationaux ${ }^{32}$, formerait possiblement l'épilogue sublime, quoique une fois encore sans lendemains, d'une histoire où la rémanence est toute révolutionnaire et où l'argument patrimonial s'inscrit dans une logique de commémoration d'un lieu, devenu immatériel, de surgissement de la parole républicaine et de l'abolition de la monarchie.

L'histoire suspendue du palais en ruines exprime donc tout autant la victoire de la République qu'une étape majeure, sur la longue durée, de séparation physique des sphères politiques et patrimoniales. Seul le ministère des Finances, dans l'intervalle de la destruction, sera parvenu en 1875, à reprendre sa place au sein du "Grand dessein " palatial. Cent ans plus tard, le difficile délogement de ce dernier accompagnera la mise en œuvre du «Grand Louvre » et le règne sans partage du patrimoine muséal au sein du palais des rois qui fut aussi le berceau perdu de la République.

III. 6 : Projet d'Auguste Sauvage et de Charles Chassin pour un Musée de la Révolution française, déposé au ministère du commerce en avril 1886

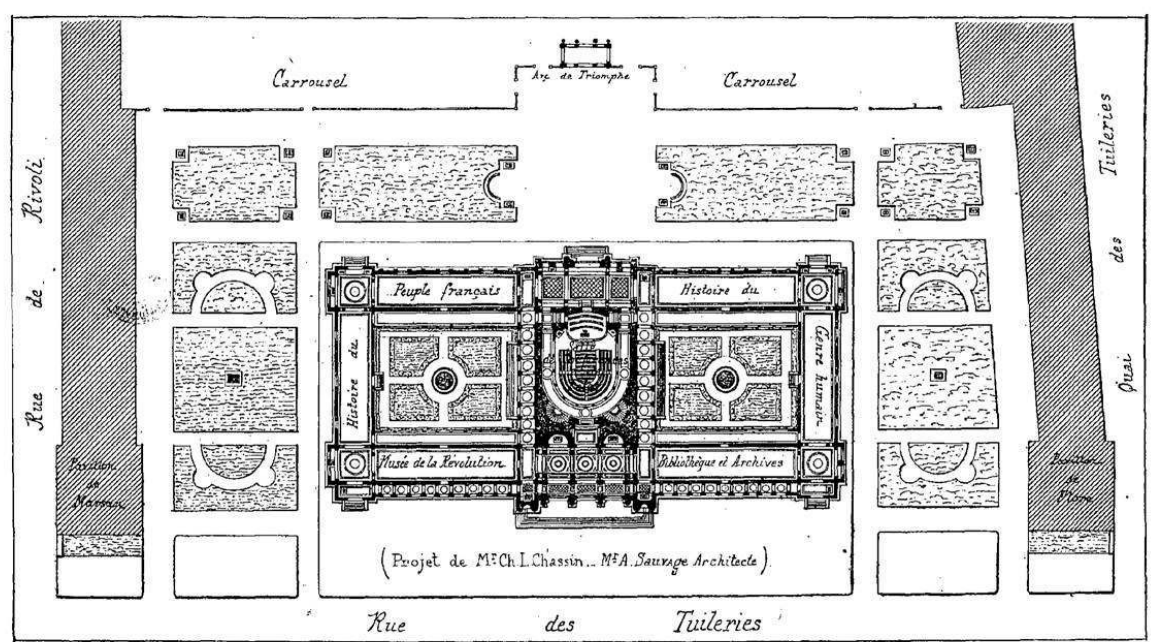

Dans Étienne Charavay, « Le Centenaire de 89 et le Musée de la Révolution », La Révolution française, 1886 (tome 12), p. 961-991, pl. hors texte 


\section{Malaise dans le Patrimoine?}

La reconstruction des Tuileries semble avoir été elle-même marquée du sceau inaugural d'une rémanence, qui est celle du vide autant que de l'accumulation, au point de n'être que l'utopie d'une restitution monumentale d'un moment précis d'une histoire architecturale et politique, non seulement entremêlée, mais uniquement dissociable par la disparition même du palais. L'inefficacité de l'argumentaire scientifique des années 1870, la controverse publique, fort difficile à restituer dans ses aspirations contradictoires, offre pour finir une grille de lecture provisoire pour éclairer de manière souterraine l'échec, peut-être temporaire, de l'émotion patrimoniale potentielle des années 2000. Un des principaux arguments mis en avant était, dans les textes du comité national pour la Reconstruction des Tuileries, d'ordre scientifique en effet, par l'évocation de la conservation d'une très abondante documentation d'archives, du plan à la photographie, permettant une opération de résurrection «fidèle ». Mais au-delà du point de vue défendu, confinant à une forme d'histoire expérimentale de l'art qui se donnerait les moyens, en grandeur réelle, de restituer une architecture perdue, de quelle " fidélité » s'agit-il ? Ironie de l'histoire, opposant à près de 140 ans d'intervalle, les partisans de deux reconstructions contradictoires : là où pendant les années 1870 , on rêvait de se débarrasser des "scories » architecturales du XIX siècle, on se propose de les faire renaitre dans leur intégralité au cours des années 2000, par la grâce des premiers monuments de la photographiques.
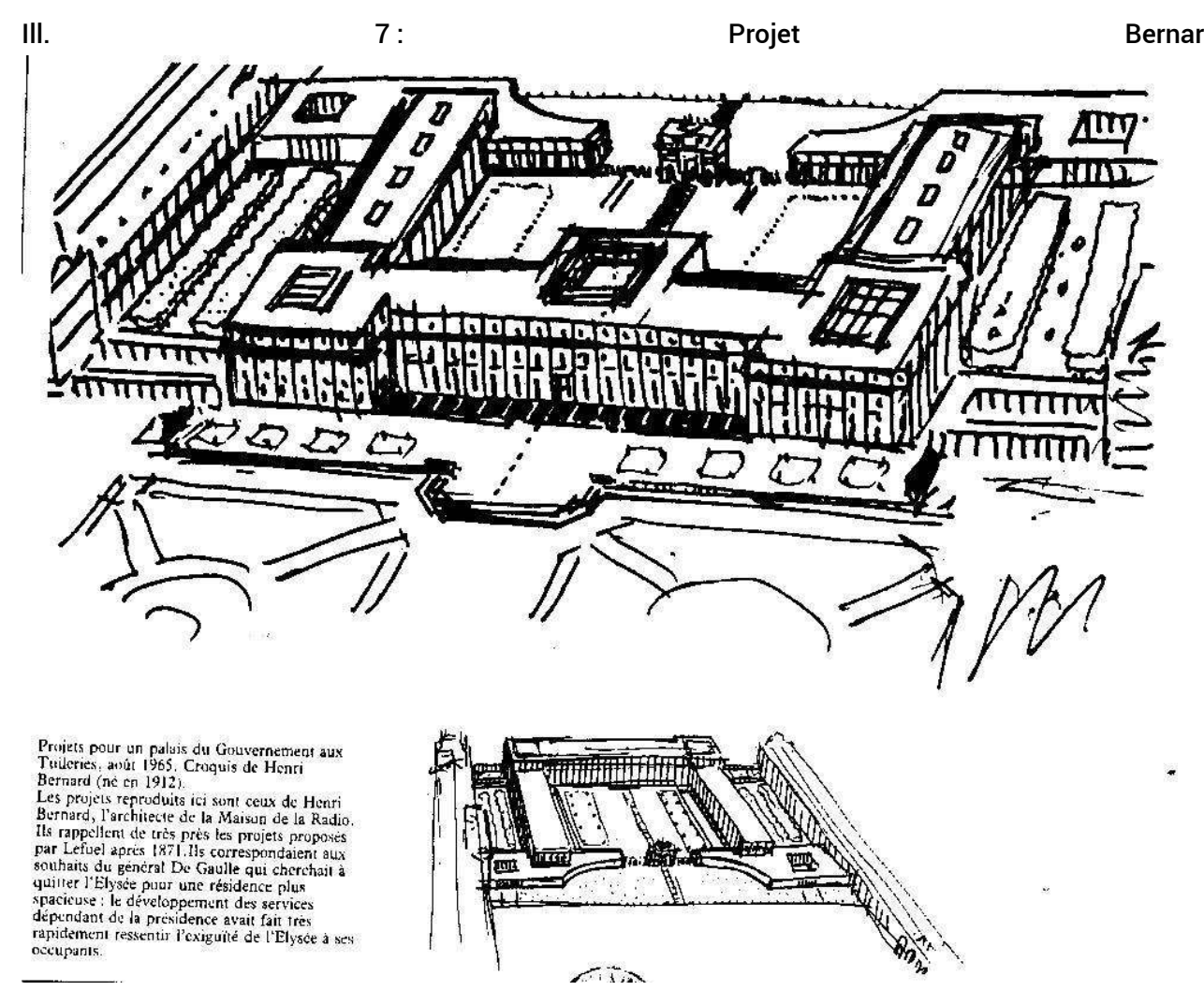

Dans Emmanuel Jacquin et Nicolas Sainte-Fare Garnot, Le Château des Tuileries, Paris, 1988

Par-delà la nostalgie d'un style, sinon d'une époque, le désir de voir renaitre le palais imaginé par Catherine de Médicis, maladroitement «rabouté » par ses successeurs 
immédiats, avant d'être alourdi par leurs lointains héritiers impériaux, reste directement connecté, par une chaîne de contre coups, à d'autres débats architecturaux et immobiliers, qui forment autant d'émotions solidaires. Hormis l'éphémère et étrange projet d'Henry Bernard de 1965, année même de la première élection au suffrage universel de son commanditaire, le général De Gaulle, d'un palais présidentiel en style moderne (ill.7), semblant mettre en adéquation la renaissance constitutionnelle du pouvoir exécutif et sa monumentalité architecturale, la dernière grande émotion patrimoniale en date aux abords des Tuileries, n'est autre, bien entendu, que la très fameuse controverse autour de l'aménagement du Grand Louvre lui-même et de sa pyramide de verre au cours des années 1980. Le désir de reconstruction du palais peut être perçu en effet comme une contre-attaque différée du projet mitterandien consistant à achever une sorte de perspective urbaine monumentale, courant du Louvre à l'Arche de la Défense, en passant par l'Arc de Triomphe. Le principal argument urbanistique repose précisément sur le rétablissement de cet axe, à partir de la façade du palais lui-même (ill.8), et non à partir de l'esplanade de la Pyramide, effectivement décalée de $5^{\circ}$, du fait de la construction indépendante des Tuileries et du Louvre, qui suit l'inflexion de la rive droite de la Seine entre ces deux ensembles.

III. 8 : Emplacement du projet de reconstruction du palais des Tuileries avec indication du décalage de perspective

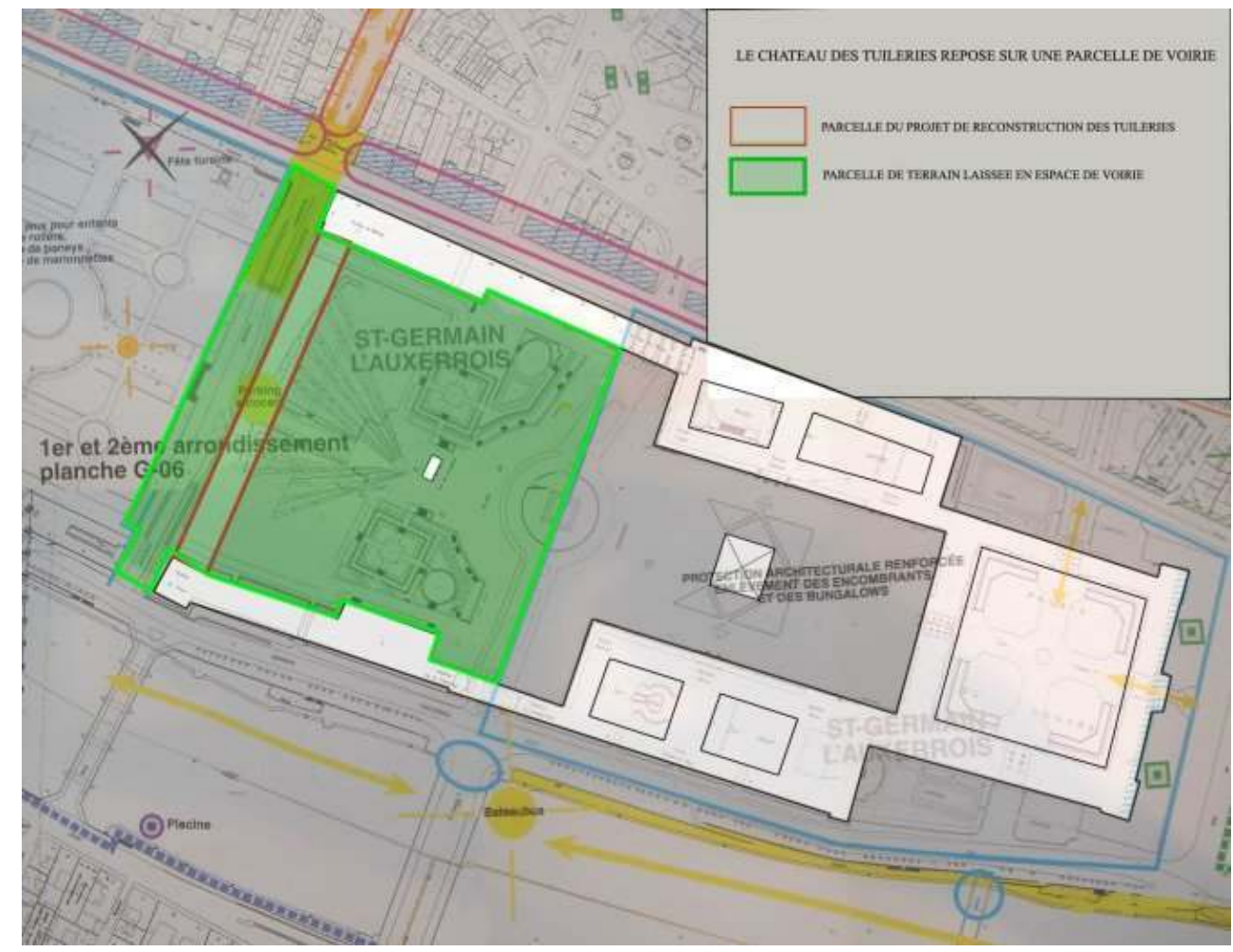

Site du comité national pour la reconstruction des Tuileries (fermé)

31 Une des conséquences de la mobilisation initiée par le comité fut de susciter, dès le départ, un débat au sein du milieu des historiens d'art, relayé notamment par le site Internet «La Tribune de l'Art», avant de faire l'objet en septembre 2010 d'un numéro entier de la revue Monumental, publication officielle du Centre des monuments nationaux. Françoise Bercé, dès les premières lignes de son éditorial, soulignait en effet combien le 
projet de reconstruction des Tuileries avait pu «susciter le trouble», au point de remettre en cause la "vulgate en matière de restauration », et de justifier pleinement la mise au point d'un tel numéro spécial ${ }^{33}$. Pour autant, le dossier, rassemblant près d'une trentaine de contributeurs, ne contient aucune étude spécifiquement consacrée aux Tuileries. Au vue du texte inaugural de Jean-Pierre Babelon, il est pourtant bien évident que cette émotion «reconstructiviste» fonctionne toujours comme une bombe patrimoniale à retardement qu'il s'agit de conjurer, ainsi qu'ont pu tenter de le faire plusieurs initiatives journalistiques et professionnelles antérieures. Après une pétition lancée en novembre 2008 contre "tous les faux en architecture ${ }^{34}$ que constituerait chaque projet de reconstruction, le comité français d'histoire de l'art, a voté une motion de protestation sur le principe même du projet des Tuileries ${ }^{35}$. Si Monumental a pu paradoxalement éviter de dédier une partie entière du dossier à l'analyse du projet de reconstruction des Tuileries, c'est sans doute du fait même de la parution exactement simultanée, au mois de septembre 2010, sous le patronage de la Cité de l'Architecture et du Patrimoine, d'une monographie historique de Guillaume Fonkenell, entièrement consacrée à l'architecture du palais disparu ${ }^{36}$. L'omniprésence au sein de l'ouvrage, sous forme de pleines pages panoramiques dépliantes, de nombreuses vues en perspective du palais reconstitué, à partir d'un support numérique en 3D, offre sur le papier la reconstruction tant désirée par les adhérents du Comité. En attendant mieux? Ce n'est pas certain.

\section{NOTES}

2. Sur l'achèvement du « Grand dessein », voir par exemple : Emmanuel Jacquin, « La réunion du Louvre aux Tuileries ", dans Louis Visconti (1791-1853), Françoise Hamon et Charles MacCallum (dir.), Paris, Délégation artistique de la Ville de Paris, 1991, p. 220-239.

3. François Macé de Lépinay, «De la restauration/restitution à la conservation des monuments? », dans Le regard de l'Histoire. L'émergence et l'évolution de la notion de patrimoine au cours du XXe siècle en France, Henry Rousso (dir.), p. 177-185.

4. Journée d'études «Les émotions patrimoniales » organisées par le ministère de la culture et de la communication, la direction de l'architecture et du patrimoine, la mission ethnologie, le CNRS, le LAHIC, à l'hôtel de Vigny en novembre 2007.

5. Guillaume Fonkenell, Le Palais des Tuileries, Paris, Éditions Honoré Clair/Cité de l'architecture et du Patrimoine, 2010, $223 \mathrm{p}$.

6. Arch. nat., F21 880 à 883 (1870-1882) et F21 2408 à 2411 (1882-1891).

7. Sur l'histoire de la direction des Bâtiments civils, et de ses « restes » archivistiques, voir en dernier lieu l'ensemble du dossier coordonné par Nadine Gastaldi, «Bâtir et orner », Livraisons d'histoire de l'architecture, $\mathrm{n}^{\circ} 21,2011$.

8. Les archives de l'agence sont partiellement conservées également aux archives nationales, sous la cote 64 AJ. Voir Emmanuel Jacquin, Cécile Souchon, Nicole Felkay, et Geneviève BrescBautier, Archives de l'Agence d'architecture du Louvre et des Tuileries (XIXe-XXe siècle), Paris, Centre historique des Archives nationales, 2006. Un bref dépouillement n'a pas permis d'identifier de dossiers conséquents permettant de dresser la symétrie des dossiers conservés en F21. 
9. Maurice Halbwachs, La Topographie légendaires des évangiles en terre sainte, Paris, PUF, 1941, 206

p.

10. Éric Fournier, Paris en ruines. Du Paris haussmannien au Paris communard, Paris, Imago, 2007, 279

p., p. 7.

11. Henri Margaine, Rapport sommaire fait au nom de la 4e commission d'initiative parlementaire sur la proposition de M. Théophile Roussel, ayant pour objet la conservation des ruines des anciennes Tuileries et de la façade de l'Hôtel-de-Ville, ainsi que des ruines des châteaux de Meudon et de Saint-Cloud, Versailles, 1871.

12. Théophile Roussel, cité par Henri Margaine, op. cit., 1871, p. 2.

13. Arch. nat., F21 880, dossier « 4-1871».

14. Arch. nat., F21 880, dossier «13-1871».

15. Arch. nat., F21 881, dossier 2.

16. Article dans La France du 31 décembre 1874 : copie dans Arch. nat., F21 881, dossier 7.

17. Arch. nat., F21 880, dossier « 7-1873».

18. Arch. nat., F21 880, dossier « 33-1873»

19. Arch. nat., F21 880, dossier «1-1872».

20. Emmanuel Jacquemin, Les Tuileries au XVIIIe siècle, Paris, Délégation à l'action artistique de Paris, 1982, 214 p. et avec Nicolas Sainte Fare Garnot, Le château des Tuileries, Paris, 1988, 224 p.

21. Guillaume Fonkenell, op. cit., p. 199-214.

22. Louis Iandoli, «The Palace of the Tuileries and Its Demolition : 1871-1883 ", dans The French Review, 76, 5, 2005, p. 986-1008; "The image of the Tuileries Palace in the French press and memoirs, 1871-1883 ", Journal of European Studies, 37, 2, 2007, p. 139-157 et, en dernier lieu, « Le palais des Tuileries : le patrimoine sacrifié », Les Cahiers du XIXe siècle, V, 2010, p. 1-15.

23. Guillaume Fonkenell, op. cit., p. 207.

24. Emmanuel Jacquemin laisse ainsi entendre que le projet de reconstruction de Freycinet de 1878 est un prétexte pour brouiller les pistes : op. cit., 1988, p. 198.

25. Arch. nat., F21 881. Ce dossier occupe la totalité d'une boîte d'archives. Ouvert en 1871, il porte un numéro d'enregistrement en 1872, puis en 1873, mais contient toutefois des documents postérieurs, jusqu'à y compris la veille du démantèlement en 1881 .

26. Arch. nat., F21 883, dossier « 1882. Enlèvement des ruines ». Il porte de manière significative, à la mine de plomb, sous ce premier intitulé, la mention "Reconstruction », ce qui laisse planer de nombreux doutes quant à sa date effective d'archivage, sans parler du fait qu'il contient des documents remontant à l'année 1876 et complémentaires du dossier précédent.

27. Arch. nat., F21 881, dossier 2.

28. Arch. nat., F21 883, dossier « 18-1879».

29. Joseph Reinach et Eugène Mir, Proposition de loi tendant à la reconstruction des Tuileries et à l'installation des deux chambres dans le même Palais. Annexe au procès-verbal de la séance de la Chambre des députés du 26 janvier 1891, Paris, 1891, 14 p.

30. Arch. nat., F21 881, dossier 6, Lettre du 21 avril 1876 au ministre des Travaux publics.

31. Pour une vue cavalière, voir Pascal Ory, Une nation pour mémoire, 1889, 1939, 1989, trois jubilés révolutionnaires, Paris, Presses de la Fondation des Sciences politiques, 1992, notamment p. 84-88. Hormis le chapitre «Le Centenaire de la Révolution aux Tuileries » dans Emmanuel Jacquin, op. cit., 1982, p. 184-191, on ne trouve aucune étude sur ces projets des années 1880. Au vue de l'ampleur du dossier documentaire disponible dans les archives des Bâtiments civils et du fait de sa complexité et de ses ramifications, nous avons renoncé à le développer ici pour le réserver pour une publication ultérieure, qui prend en charge d'autres tentatives de patrimonialisation et de « mise en musée » du moment révolutionnaire.

32. Soit l'équivalent d'une pleine boîte d'archives : Arch. nat., F21 2410. 
33. François Bercé, "Editorial ", dans «Achèvement/Restitution/Reconstruction ", Monumental, 2010 (1), p. 4.

34. Pour le texte de la pétition, voir:: http://www.paperblog.fr/1304770/une-petitioncontre-les-idees-de-reconstruction/

35. http://www.cfha-web.org/actualite/motion_AG_2009_1.htm : “ Le Comité s'étonne qu'un tel projet puisse faire abstraction du passage du temps et de ses conséquences. Plus de cent vingt cinq ans se sont écoulés depuis la décision d'enlèvement des ruines du château prise par le gouvernement de la IIIe République. L'immense espace ouvert créé par cette décision est désormais inscrit dans le paysage de la capitale. Il en a influencé de façon déterminante l'urbanisme au cours du XXe siècle, notamment pour les développements accordés à la perspective centrale, courant désormais de la Pyramide de Peï jusqu'à l'Arche de la Défense. Il s'agit là d'une composante essentielle du patrimoine de Paris, appréciée du public et universellement reconnue. Le Comité regrette enfin, qu'au moment où des interventions urgentes sont indispensables sur tant de monuments historiques célèbres et encore existants, on puisse envisager de détourner de façon irresponsable compétences, temps et moyens au profit d'un projet aussi discutable. Il est faux de prétendre que celui-ci pourrait être réalisé sans participation de financements publics. En revanche, les véritables objectifs d'éventuels investisseurs privés sur un tel emplacement au cœur de la capitale, ne sauraient tromper personne. Pour ces raisons, le comité français d'histoire de l'art proclame son opposition complète et unanime au projet de reconstruction du château des Tuileries."

36. Guillaume Fonkenell, op. cit.

\section{RÉSUMÉS}

La rumeur, les réactions et les débats suscités, au sein du monde de la Culture et du patrimoine pour l'essentiel, au cours des années 2000, par un projet de reconstruction du palais des Tuileries constituent une forme d'émotion patrimoniale "en puissance», qui semble désormais en sommeil. Portée par l'académie du Second Empire et par un comité national dédié à partir de 2004, cette émotion patrimoniale avortée s'est très vite révélée être également une émotion pour le Patrimoine, en tant que champ professionnel et scientifique, dans le sens où la dimension, en apparence inédite, d'une telle revendication a fait (re)surgir une série de débats théoriques et idéologiques fondamentaux quant à la politique de conservation des monuments historiques et quant aux principes qui guident la notion même de « restauration » du patrimoine architectural en France depuis plus d'un siècle. À partir d'une analyse des principaux arguments convoqués par les partisans de la reconstruction - et tout particulièrement l'idée d'une "rémanence » supposée du lieu au cœur de la capitale - cet article se propose de revenir sur l'histoire complexe de la disparition et de la non-reconstruction d'un monument qui fut, sans discontinuité, le siège du pouvoir exécutif suprême, d'octobre 1789 à septembre 1870. Si le 24 mai 1871, le Palais des Tuileries a fait partie des nombreux bâtiments civils parisiens incendiés par la Commune de Paris, sa démolition définitive revient cependant à la toute jeune République, et, plus particulièrement à l'action décisive de l'éphémère "ministère des Arts ", entre novembre 1881 et janvier 1882. La mise en œuvre, entre 1884 et 1888, au lendemain de la destruction effective en 1883, du projet de reconstruction d'un nouveau Palais, dédié à un «musée de la Révolution 
française ", donne la mesure du refoulement et de la rémanence dont le lieu a pu faire l'objet. L'échec récurrent de ce qui apparaît, pour finir, comme une émotion "reconstructiviste", permet de saisir un écheveau monumental complexe où patrimoine et politique s'incarnent mutuellement l'un par l'autre et l'un en l'autre.

Monument politique du XIX ${ }^{e}$ siècle, symbole de la continuité de l'État en dépit des fréquents changements de régime, le Palais des Tuileries fut comme la victime sacrificielle d'un régime républicain résistant à s'incarner en un corps exécutif fort. Les ruines du palais, comme les projections dont elles ont pu faire l'objet après leur disparition, offrent la possibilité d'écrire, en parallèle et en miroir, une histoire politique du patrimoine qui puisse être simultanément un histoire patrimoniale du politique.

The rumours, reactions and debates generated, essentially within the world of culture and heritage during the first ten years of our century by a project to reconstruct the Tuileries Palace represent a form of 'potential' heritage emotion, which seems however, to have died down now. Supported by the Académie du Second Empire and by a special national committee founded in 2004, this aborted heritage emotion rapidly emerged as an emotion for the heritage, considered as a professional and scientific domain. The apparently unanticipated dimension of this suggestion brought forth a series of fundamental theoretical and ideological debates concerning the policies of monument conservation and the principles which guide the very notion of 'restoration' of the architectural heritage in France over the last century. Analysing the main arguments put forward by the partisans of the reconstruction of the palace, and in particular the idea of a supposed 'remanence' at the heart of Paris, this article proposes to return to the complex story of the disappearance and non-reconstruction of a monument which was, without interruption, the seat of the supreme executive power, from October 1789 to September 1870 . The Tuileries Palace was one of the many civil buildings in Paris destroyed by fire from 24 May 1871 at the end of the Commune, but its final and definitive demolition was the work of the young Third Republic and, more particularly, the decisive action of the ephemeral 'Ministry of Arts', between November 1881 and January 1882. The proposal, between 1884 and 1888, after the effective destruction in 1883 of the remains of the palace, of a project for the construction of a new palace, dedicated to a 'Museum of the French Revolution', gives some idea of the psychological repression and of the remanence associated with the site of the palace. The recurrent failure of what emerged, in the end, as a 'reconstructivist' emotion, allows for the analysis of a complex monumental web in which heritage and politics mutually incarnate each other, one by the other and one in the other.

As a political monument of the nineteenth century, as a symbol of the continuity of the State, despite changing regimes, the Tuileries Palace was a sort of sacrificial victim for a republican regime which was anxious not to establish too powerful an executive body. The ruins of the palace, like the projections that these ruins were the object of after their disappearance, offer the possibility of writing, in parallel and in mirror form, a political history of heritage which is also a heritage history of politics.

Die Gerüchte, die Reaktionen und die Debatten, die im Laufe der Jahre ab 2000 hauptsächlich in den Kreisen der Kultur und des Denkmalschutzes durch ein Projekt des Wiederaufbaus des Tuilerienpalastes erregt wurden, erwiesen sich als eine Art potentieller émotion patrimoniale, die heutzutage ausgelöscht scheint. Von der Akademie des Second Empire und einem ab 2004 dazu dedizierten Nationalkomitee unterstützt, wurde diese gescheiterte émotion patrimoniale bald eine Emotion für den Denkmalschutzdienst selber als professionelles und wissenschaftliches Feld. Sozusagen ließ die anscheinend neuartige emotionale Dimension eines solchen Anspruchs eine Reihe von theoretischen und ideologischen Debatten wieder aufleben. Sie betrafen die Politik des Denkmalschutzes sowie die Leitlinien des Begriffs von Restaurierung des Bauerbes in Frankreich seit über einem Jahrhundert. Auf der Basis der Analyse der hauptsächlichen Argumente, die von 
den Anhängern des Wiederaufbaus vorgebracht wurden, - insbesondere die Idee, dass diesem Ort im Herzen der Hauptstadt eine anzunehmende Kontinuität verliehen sei -, nimmt sich der Artikel vor, auf die komplexe Geschichte des Verschwindens und des Nicht-Wiederaufbaus eines Denkmals zurückzukommen, das von Oktober 1789 bis September 1870 ununterbrochen der Sitz der obersten Exekutivgewalt war. Der Tuilerienpalast gehört zwar zu den vielen Pariser Zivilgebäuden, die am 24.Mai 1871 von der Pariser Kommune in Brand gesteckt wurden. Dessen endgültiger Abriss kann aber der ganz jungen Republik zugeschrieben werden, beziehungsweise der folgenschweren Entscheidung des nur kurzfristig bestehenden ministère des Arts ( Ministerium für Künste) zwischen November 1881 und Januar 1882. Zwischen 1884 und 1888, kurz nach der effektiven Zerstörung im Jahre 1883, entstand ein Projekt für den Wiederaufbau des Palastes, der als ein Museum der französischen Revolution fungieren sollte. Es zeugt von dem Ausmaß der remanenten Widerstände, die dieser Ort erregen konnte. Das immer wiederkehrende Scheitern davon, was sich schließlich als ein emotionales Bedürfnis wiederaufzubauen oder eine émotion „reconstructiviste “ erweist, stellt einen komplexen Zusammenhang vor Augen, wo Denkmalerbe und Politik sich gegenseitig verkörpern, das eine durch das andere und das eine in dem anderen. Der Tuilerienpalast, politisches Gebäude des 19. Jahrhunderts, auch Symbol der Kontinuität des Staates trotz wechselhafter Regierungsformen, wurde von dem republikanischen Regime aufgeopfert, welches zögerte, sich als starke vollziehende Macht zu behaupten.

\section{AUTEURS}

\section{VINCENT LEMIRE}

Vincent Lemire, ancien élève de l'ENS de Fontenay/Saint-Cloud, est maître de conférences en histoire contemporaine à l'Université Paris-Est Marne-la-Vallée et membre du laboratoire « Analyse comparée des pouvoirs » (EA 3350). Ses recherches portent sur Jérusalem et le ProcheOrient contemporain, l'histoire environnementale et la patrimonialiasation urbaine. Il a publié en 2010 La soif de Jérusalem. Essai d'hydro-histoire (1840-1948), Paris, publications de la Sorbonne. Yann Potin, ancien élève de l'ENS de Fontenay/Saint-Cloud, archiviste-paléographe, est chargé d'études documentaires aux Archives nationales et membre associé du IIAC, équipe "Anthropologie de l'écriture » (UMR 8588). Ses recherches portent sur l'histoire du patrimoine et tout particulièrement sur l'histoire des institutions d'archives depuis le Moyen Âge. 